Musées, Patrimoine et Culture scientifiques et techniques

$141 \mid 2012$

mai - juin 2012

\title{
Diffuser la culture scientifique et technique au Maroc
}

Diffusing scientific and technical culture in Morocco

\section{Afaf Mikou et Aziz Bensalah}

\section{OpenEdition}

\section{Journals}

Édition électronique

URL : http://journals.openedition.org/ocim/1071

DOI : 10.4000/ocim. 1071

ISSN : 2108-646X

Éditeur

OCIM

Édition imprimée

Date de publication : 1 mai 2012

Pagination : 24-32

ISSN : 0994-1908

Référence électronique

Afaf Mikou et Aziz Bensalah, «Diffuser la culture scientifique et technique au Maroc », La Lettre de I'OCIM [En ligne], 141 | 2012, mis en ligne le 01 mai 2014, consulté le 19 avril 2019. URL : http:// journals.openedition.org/ocim/1071; DOI : 10.4000/ocim.1071 
le pôle non institutionnel, constitué par un mouvement associatif centré sur les sciences et les techniques, et dont le noyau dur est un ancien acteur du PCST.

Une autre bonne nouvelle est l'inscription (implicite) de la CST dans la toute nouvelle constitution marocaine, adoptée en juin 2011. L'article 33 de cette constitution stipule en effet qu' « il incombe aux pouvoirs publics de prendre toutes les mesures appropriées en vue de... faciliter l'accès des jeunes à la culture, à la science, à la technologie, à l'art, au sport et aux loisirs, tout en créant les conditions propices au plein déploiement de leur potentiel créatif et innovant dans tous ces domaines. Il est créé à cet effet un Conseil consultatif de la jeunesse et de l'action associative ».

Diffuser la CST au Maroc se présente donc aujourd'hui sous de meilleurs auspices, mais les difficultés, voire les blocages, restent nombreux et la pleine mobilisation souhaitable n'est toujours pas au rendezvous.

\section{Problématique de la CST au Maroc}

Le contexte général marocain est difficile (4) (5): de manière succincte, on peut dire qu'il reste marqué aujourd'hui, malgré de réels efforts et des politiques ambitieuses de correction, par un fort taux d'analphabétisme, de grandes disparités régionales, sociales et de genre, et des systèmes d'éducation globalement peu performants voire défaillants s'agissant du milieu rural.

Quelques chiffres significatifs du contexte marocain

Population en 2010 : 32 millions d'habitants dont $42 \%$ rurale et $28 \%$ de moins de 15 ans

Taux d'analphabétisme adulte : $44 \%$ avec $28 \%$ des hommes et $50 \%$ des femmes

Taux de scolarisation : primaire $95 \%$, collèges $75 \%$, lycées $50 \%$

Taux de scolarisation des filles en milieu rural : primaire $92 \%$, collèges $46 \%$, lycées $14 \%$

Écoles rurales raccordées au réseau électrique : $40 \%$

Néanmoins, ce contexte est potentiellement favorable à la diffusion de la CST. Il suffit de souligner l'existence d'un fort taux de scientifiques, universitaires et non universitaires, bien répartis géographiquement, face à une population bénéficiant, en très grande majorité, des avancées scientifiques et technologiques spectaculaires mais dont la compréhension lui échappe. À titre d'exemple, la télévision par satellite et le téléphone portable font partie du quotidien de la majorité des citoyens marocains, y compris dans les

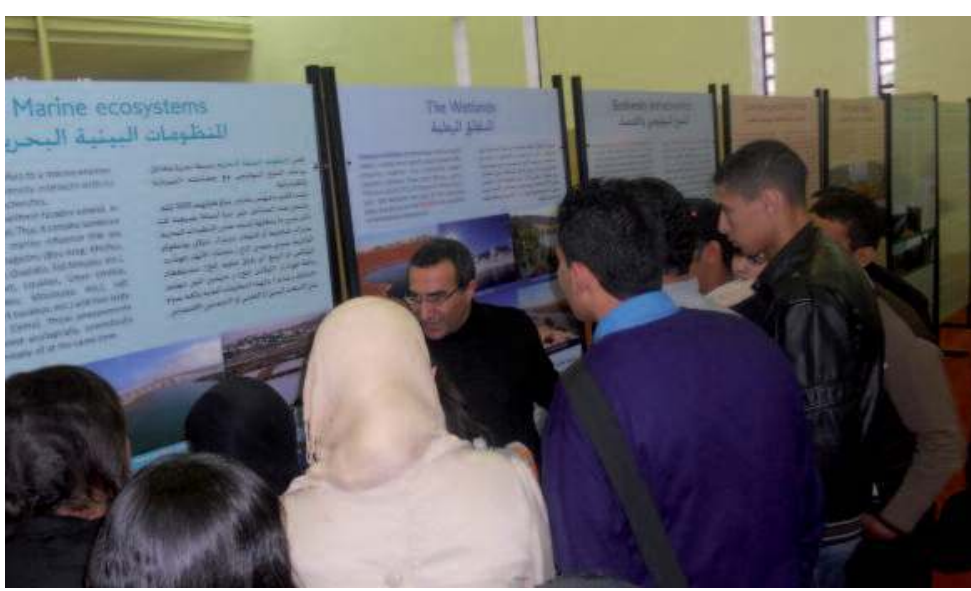

L"exposition Connaissance de la Biodiversité marocaine présentée à Casablanca en décembre 2011 dans l'espace "Les jeunes, la science et la technologie » du Salon de la Recherche, de l'innovation et de la Technologie, Medinnova 2011. ๑) Club Science Citoyenne, RNCST/Afaf Mikou

régions rurales les plus reculées. De même, les techniques d'imagerie échographiques en obstétrique, aujourd'hui largement répandues, ne manquent pas de susciter l'étonnement et le questionnement non seulement des patientes auscultées mais aussi de leurs familles lorsqu'on leur révèle le sexe de leur futur enfant.

Cependant, diffuser la CST au Maroc nécessite de prendre en considération l'état contrasté de son paysage actuel ${ }^{(6)}$, à commencer par quelques particularités de la société marocaine, partagées avec d'autres pays du monde arabe, notamment ceux du Maghreb.

En premier lieu, il faut constater la diversité voire l'opposition des publics concernés : éduqué/non éduqué, urbain/rural, jeune/âgé, aisé/de condition modeste, langue d'usage/ langue d'éducation.

En second lieu, bien que la science et la technologie bénéficient d'une opinion, en général, très positive pour des raisons historiques et culturelles (âge d'or de la civilisation arabo-musulmane, valeur de l'apprentissage et de la transmission du savoir en Islam) et vu l'essor technologique expérimenté au quotidien, ceci est contrebalancé par le fort taux de chômage parmi les diplômés en sciences. "La science ne nourrit pas son homme » est une idée assez répandue au sein de la population marocaine.

En troisième lieu, à l'instar des sociétés européennes, la société marocaine est de plus en plus une société de loisirs et la science entre difficilement en compétition avec l'offre faite dans ce domaine, notamment auprès des jeunes. 
En dernier lieu, fait le plus important, loin de la crise de confiance connue par leurs homologues occidentaux, les scientifiques marocains font face à une crise d'indifférence.

Malgré ce constat, il est intéressant d'observer, ainsi que nous l'avons déjà mentionné, l'existence d'un mouvement associatif fort et très engagé dans la diffusion de la CST. À l'opposé, s'agissant des autres acteurs de CST, potentiels ou réels, on observe, pour certains, une présence au mieux insuffisante et pour d'autres, une quasi-absence.

Ainsi, les institutions publiques en charge des politiques scientifique et technologique et/ou de la formation à différents niveaux, sont peu nombreuses à intervenir. Elles se réduisent dans la pratique au MESRSFC (ministère de l'Enseignement supérieur, de la Recherche scientifique et de la Formation des cadres), à l'Académie Hassan II des Sciences et Techniques et au Centre national pour la Recherche scientifique et technique (CNRST). Le MESRSFC finance annuellement l'organisation d'une «Semaine de la science » tournante dans les universités. L'Académie Hassan II des Sciences et des Techniques organise chaque année, à l'échelle nationale, une manifestation intitulée "Les jeunes et la science " se traduisant par des conférences-débats et des démonstrations dans plusieurs établissements, allant du primaire aux universités. Le CNRST, quant à lui, a choisi de constituer un réseau national de clubs universitaires, présenté dans la suite de cet article. Par ailleurs, on déplore toujours, au Maroc, l'absence de sites de science (musées, cités ou centres); cependant, cette nécessité est reconnue de manière unanime et fait même l'objet d'un débat national (en attendant la prise de décision !) quant à la nature et la taille de ces sites.

Parmi les acteurs potentiels dont l'absence est fortement pénalisante pour le développement de la CST au Maroc, les médias viennent certainement au premier rang. Pour l'heure, on ne dénombre aucune émission radiophonique ou télévisuelle consacrée à la diffusion de la CST. De même, aucune revue de vulgarisation scientifique n'est éditée au Maroc et la diffusion des revues étrangères en vente, principalement françaises, est certainement insignifiante. Enfin, on déplore l'absence de journalistes scientifiques dûment formés ou reconnus en tant que tels, à même de couvrir les manifestations scientifiques ou d'effectuer des reportages.

Sur le plan politique, s'agissant de la CST, il n'existe jusqu'à présent ni rapport émanant du Parlement

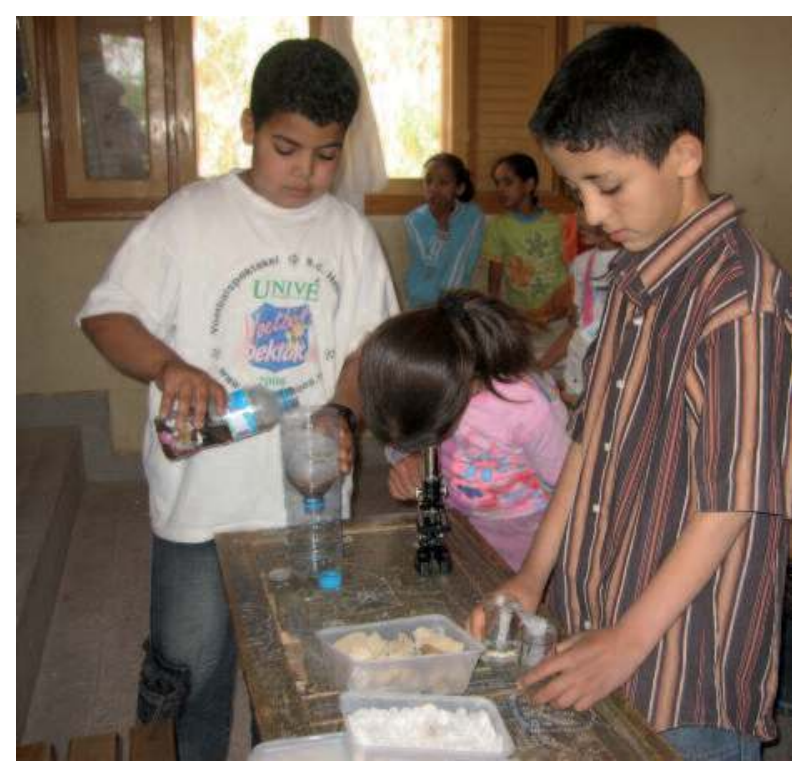

L'atelier scientifique Construisons une station d'épuration, réalisé dans une école de la région de Zagora en avril 2009. () Club Science Citoyenne, RNCST/Afaf Mikou

national, ni traces évidentes dans les programmes des partis politiques. De même, dans les sphères gouvernementales, le niveau de CST au sein de la population n'est toujours pas reconnu comme un facteur important du développement socio-économique.

Finalement, à la suite de ce tableau complexe avec bien des aspects négatifs, il apparaît que mettre en chantier la CST au Maroc constitue un sérieux défi. Pour autant, le partage des savoirs, facteur de justice et d'intégration sociales, est à même de mobiliser beaucoup de forces sociales créatives. Les deux exemples présentés ci-dessous traitant de l'actuelle implication d'universitaires marocains et de leur investissement en milieu rural marocain en font, à notre sens, la démonstration.

\section{Le RNCST : un outil au service de la diffusion de la CST au Maroc}

Au Maroc, le Réseau national pour la promotion de la diffusion de la culture scientifique et technique (RNCST), créé en mars 2008 par le CNRST, a pour vocation de regrouper les structures universitaires marocaines œuvrant dans le domaine de la CST. Historiquement, il s'agit de la rencontre entre la volonté de plusieurs acteurs de la CST, universitaires et lauréats du PCST, de se constituer en réseau (7) et le rôle de fédérateur et de facilitateur que le CNRST entend jouer dans tout domaine contribuant à renforcer le potentiel scientifique et technique du 
royaume. L'objectif général déclaré du RNCST est le « développement de la capacité du citoyen marocain à comprendre et à se saisir des enjeux de société impliqués par les avancées scientifiques, techniques et industrielles». Pour ce faire, il s'appuie sur ses membres, clubs universitaires répartis dans plusieurs universités marocaines et porteurs de différentes thématiques.

Le RNCST offre à ses membres la possibilité d'un financement de leurs projets de CST, une ouverture sur l'international par le biais d'accords de coopération avec des réseaux CST étrangers et la possibilité de se porter candidats à des appels à projets internationaux. Il est dirigé par un comité de pilotage constitué par cinq universitaires dont le rôle est d'assurer une animation et un suivi des activités. Ce comité statue sur les demandes d'adhésion et valide les projets de CST soumis. Son fonctionnement a minima est assuré par l'octroi d'un budget annuel par le CNRST. Cependant, nombre d'actions, individuelles ou collectives, sont financées par d'autres moyens : projets internationaux, conventions avec des organismes nationaux ou étrangers, subventions publiques ou privées. Ouvertes au grand public, les activités du RNCST ciblent de manière particulière les jeunes directement ou via les réseaux scolaires et universitaires. Force de réflexion, le RNCST œuvre à la définition et à l'adoption d'une politique nationale de CST.

Les objectifs qui sous-tendent ces actions vont de l'apprentissage informel de connaissances scientifiques et techniques à la mise en débat de questions de société soulevées par les avancées scientifiques et techniques, en passant par l'extension et l'approfondissement de la culture scientifique, l'acquisition ou la réappropriation de la méthode scientifique. De même, la sensibilisation via la démonstration et l'argumentation scientifiques est souvent présente, par exemple dans les domaines de l'environnement et de la santé. Dans la pratique, l'approche de médiation et de communication scientifiques prend en compte autant que possible la ou les spécificités de l'audience concernée. Les outils et modes d'expression sont nombreux : ateliers, expositions interactives, films et conférences débats, cafés scientifiques, télévision et Internet, conférences, sorties thématiques... et sont choisis et adaptés en fonction du public concerné.

Le RNCST compte aujourd'hui 8 clubs (voir encadré ci-dessous) répartis dans 7 universités. Certaines de ses activités annuelles ont acquis une dimension internationale : le Festival du film scientifique de Marrakech organisé par le Club de journalisme scientifique et la Traversée de l'Oriental organisée par l'Association Nature et Patrimoine. Le Club Science citoyenne organise chaque année un concours «Sciences et expériences » ouvert aux étudiants casablancais et a l'intention de l'étendre graduellement à tout le Maroc. Le muséum d'Histoire naturelle de Marrakech organise chaque année des Journées scientifiques où, aux côtés des conférences scientifiques, les jeunes, lycéens et étudiants, sont invités à découvrir ses collections de faune et de flore et à participer à

\section{Les clubs et partencires du RNCST}

\section{Clubs}

- Club de Journalisme Scientifique (Université

Cadi Ayyad, Marrakech)

- Club Tous pour la Science (Université Ibn Tofail, Kénitra)

- Club Science citoyenne (Université Hassan II Ain Chock, Casablanca)

- Muséum d'Histoire naturelle de Marrakech

(Université Cadi Ayyad, Marrakech)

- Club Scientifique et Culturel Ibn Zohr

(Université Ibn Zohr, Agadir)

- Association Nature et Patrimoine (Université

Mohamed 1er, Oujda)

- Club Hélios Santé et Environnement (Université Hassan II - Ben M'sik, Mohammedia)

- Association Méditerranéenne pour la Vulgarisation des Sciences (Université Abdel Malek Essaadi,

Tétouan)

\section{Partenaires}

- La réunion des Centres de Culture Scientifique, Technique et Industrielle (CCSTI, France)

- L'Institut pour la Recherche et le Développement (IRD, France)

- CNRS-Images (France)

- Le Réseau Romand Science et Cité (RRSC, Suisse)

- Les Instituts Français au Maroc

- Le British Council au Maroc

- L’Association des Enseignants des Sciences de la Vie et de la Terre (AESVT, Maroc)

- L'Association Marocaine des Petits débrouillards (AMPD, Maroc) 


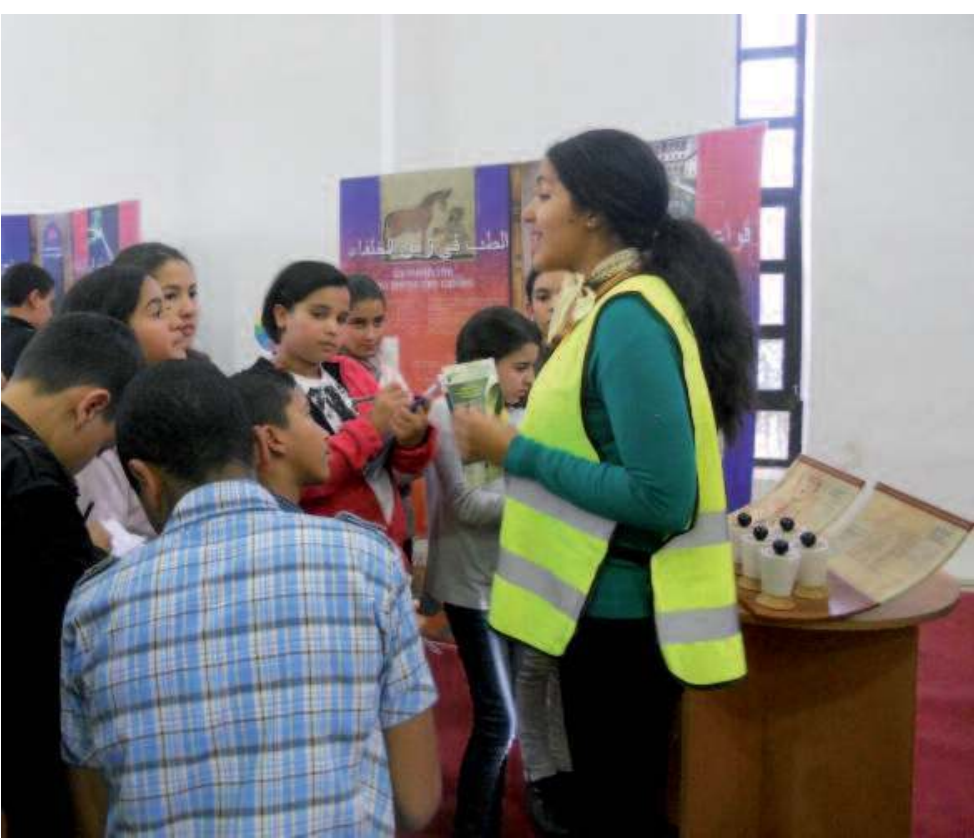

Découverte des sciences arabo-musulmanes à travers l'exposition Quand les sciences parlent arabe présentée à Casablanca en décembre 2011 dans l'espace « Les jeunes, la science et la technologie » du Salon de la Recherche-Développement, de I'Innovation et de la Technologie, Medinnova 2011. (c) Club Science Citoyenne, RNCST/Afaf Mikou

des ateliers de classification des espèces. Le Club scientifique et culturel de l'université Ibn Zohr à Agadir, qui possède une collection intéressante de météorites du Sud du Maroc proposée en exposition itinérante, rencontre beaucoup de succès dans ses activités d'initiation à l'astronomie. Il organise régulièrement des séances d'observation du ciel dans la région Sud. Les activités d'astronomie, populaires au Maroc, sont assurées notamment par le Réseau des astronomes amateurs marocains (RAAM).

Le RNCST agit aussi collectivement dans le cadre de projets ou d'événements impliquant plusieurs clubs, les derniers en date étant le projet Transavia Solid'Air 2011 pour l'environnement ou encore l'organisation et l'animation de l'espace « Les jeunes, la science et la technologie » du Salon de la Recherche-Développement, de l'Innovation et de la Technologie, Medinnova 2011 Casablanca. Du point de vue de l'impact, malgré l'absence d'un suivi chiffré et détaillé des activités de tous les clubs, nous estimons que les événements cumulés du RNCST en 2011 ont drâné plusieurs milliers de visiteurs et/ou participants.

À ce titre, l'une des activités rencontrant le plus de succès est l'initiation à l'histoire des sciences arabomusulmanes à travers l'exposition Quand les sciences parlent arabe. Cette exposition bilingue français-arabe, réalisée par Centre Sciences d'Orléans, CCSTI de la région Centre, et le Centre français de Culture et de Coopération du Caire (CFCC) et attribuée au CNRST par le MAAE, permet au public marocain de découvrir les avancées scientifiques et techniques réalisées du VIIIe au XVe siècle dans le monde arabomusulman. Elle montre avec brio le souci constant des savants de l'époque de satisfaire à des demandes sociales et de partager leurs connaissances. De plus, judicieusement construite autour de sept thèmes (observer, mesurer, calculer, expérimenter, soigner, construire et transmettre) et de dix-huit manipulations interactives associées, elle est très adaptée à l'exposition de la méthode scientifique et de la relation sciences et techniques.

L'évocation de cette exposition nous amène à parler brièvement de la réaction du public marocain (en fait, des publics) aux différentes manifestations auxquelles il est convié. S’il s’agit, en général, d'un public montrant un réel intérêt, il est indubitable qu'il a des préférences, traduites par un plus grand engouement et une meilleure réactivité, par rapport aux thématiques des activités proposées. Ainsi, en est-il des expositions renvoyant à des préoccupations nationales (santé, environnement, nouvelles technologies favorables au développement du Maroc...). Quant à l'exposition Quand les sciences parlent arabe, les sentiments de fierté et de nostalgie à l'égard d'un passé glorieux sont nettement présents. Par ailleurs, lors des cafés scientifiques et des films-débats, il est intéressant de noter que la parole se libère petit à petit pour devenir parfois... incontrôlable!

Enfin, il convient de signaler que le RNCST organise aussi ses événements propres comme, en octobre 2009, le premier colloque international sur la CST au Maroc (8). De même, chaque année, un séminaire tournant au niveau des clubs est consacré à l'ouverture sur l'environnement scientifique et socio-économique régional et à la gestion des affaires internes.

\section{L'exemple du milieu rural : relever le défi du développement humain}

Au Maroc, l'Initiative nationale de Développement humain (INDH) ${ }^{(9)}$ est un projet d'envergure nationale adopté en reconnaissance du rôle stratégique joué par le développement humain durable dans le développement économique et social. Parmi les objectifs annoncés en vue de l'instauration de cette dynamique de développement, figurent la réduction 


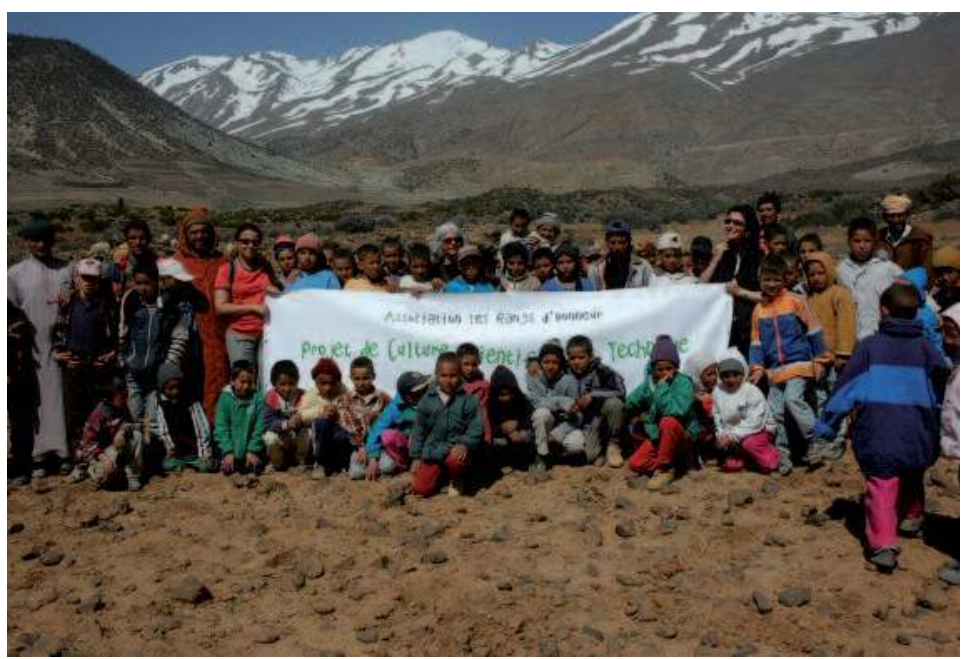

Les participants à l'atelier Sel iodé indispensable pour la santé organisé en avril 2006 au village Timjilt, près du mont Bou lblane dans la région de Taza. (c) Association les Rangs d'Honneur/Driss El Ouazzani

de la pauvreté et de l'exclusion sociale, la reconnaissance de la dignité de l'homme et le renforcement de la cohésion sociale.

À ce titre, vu les grandes disparités de revenus, d'infrastructures et de conditions de vie et d'éducation qui l'opposent au milieu urbain, le milieu rural marocain a bénéficié d'un des quatre programmes prioritaires de cette initiative. Ce programme ambitieux, intitulé « Programme de lutte contre la pauvreté en milieu rural », s'appuie sur ces quatre composantes :

- activités génératrices de revenus ;

- soutien à l'accès aux équipements et services sociaux de base ;

- soutien aux actions d'animation sociale, culturelle et sportive ;

- soutien au renforcement de la gouvernance et des capacités locales.

En fait, au cœur de ce programme, il y a la reconnaissance de l'urgence du développement des compétences humaines en matière d'acquisition de savoir et de savoir-faire. La diffusion de la CST y a donc toute sa place et invite à trouver des modes d'expression appropriés.

Dans cette optique, une manière originale d'investir le milieu rural a été développée dans le cadre d'un programme deux fois lauréat du PCST (en 2006 et en 2009) (10). Bien que déjà exposé par ailleurs (11) (12), nous rappelons ici l'essentiel de ce programme avant de préciser son évolution et son développement dans le cadre du RNCST.

Mené en étroite collaboration avec l'Association les Rangs d'Honneur à l'occasion de campagnes médicales et sanitaires gratuites organisées par cette ONG et draînant une large population rurale, ce programme, toujours d'actualité, cible les jeunes à travers des ateliers de sensibilisation à des problématiques prédominantes dans la région visitée (maladies hydriques, troubles dus aux carences en iode...). Ces ateliers s'appuient sur l'utilisation combinée d'outils scientifiques et de modes d'expression ludiques et pédagogiques. L'objectif affiché est d'aboutir à une auto-prévention et une amélioration de la qualité de vie de ces populations via une éducation les amenant à changer les mauvais gestes en usage.

Concrètement, dans de tels ateliers, l'animateur scientifique provoque les réactions des jeunes en les interpellant sur des situations de leur quotidien. Par exemple, une jeune fille se voit proposer de l'eau souillée. Bien évidemment, elle refuse de la boire en avançant qu'elle n'est pas « claire ». Par la suite, avec l'aide de l'animateur, cette jeune fille traite l'eau souillée sur un dispositif expérimental de filtration progressive et obtient au final une eau limpide qu'elle accepte de boire. L'animateur sort alors son microscope et montre que l'eau, bien que claire, est contaminée par des bactéries. Le traitement par l'eau de javel élimine alors ces bactéries. On fait ainsi la démonstration que cette étape de chloration, recommandée par le ministère de la Santé lors des campagnes de sensibilisation mais très souvent négligée par les jeunes qui vont au puits (en majorité des filles), est indispensable pour rendre l'eau potable. La jeune fille participant à l'expérience en prend définitivement conscience et sera à même de transmettre le message de prévention sanitaire. Par la suite, l'animateur, ayant acquis la confiance du public présent, est alors à son aise pour affirmer que d'autres particules non observables, même au microscope, peuvent souiller l'eau (métaux lourds...) et que la seule façon de la préserver totalement est de protéger son environnement en s'interdisant de jeter des produits contaminants tels que piles et autres déchets électroniques.

L'atelier précédent, intitulé «Construisons une station d'épuration », a remporté un franc succès de même qu'un deuxième atelier traitant des carences en iode dues au sel gemme des montagnes (11) (12).

Depuis 2006, le programme susmentionné dédié au monde rural a bénéficié à plus de 10000 jeunes et, à travers eux, à leurs parents souvent analphabètes. Pour une grande part, il a valu à son initiatrice, coauteure de cet article, le prix PURKWA, grand prix de l'Académie des Sciences française pour l'alphabétisation des enfants de la planète (12)(13). 
Le lac Zima, zone humide protégée, d'une superficie de 558 hectares, est situé à une dizaine de kilomètres à l'Ouest de la ville de Chemmaia (Province de Safi, 70 km à l'ouest de Marrakech, 365 m d'altitude). C'est un site Ramsar (15), eu égard à sa richesse ornithologique, dont la biodiversité est toutefois mise en danger par une activité socio-économique centrée sur la production de sel.

La journée du 4 juin 2011 a été dédiée à la sensibilisation à la protection de la faune et de la flore des environs de Chemmaia, en général, et du lac Zima, en particulier. Cette action s'est déroulée au Collège du 30 juillet de la ville de Chemmaia et s'est appuyée sur l'organisation de quatre ateliers éducatifs au profit de 180 collégiens en présence de professeurs et d'administratifs du collège ainsi que du directeur de l'établissement.

Le déroulement des ateliers a été conçu de manière à permettre aux collégiens y participant d'acquérir une réelle autonomie vis-à-vis de la protection de leur environnement, notamment celui du lac Zima, et de pouvoir sensibiliser les populations rurales environnantes, en général non éduquées.

D’une durée moyenne de 30 minutes chacun, les quatre ateliers ci-dessous se sont déroulés en parallèle et en roulement :

- atelier 1 : Présentation générale du site Lac Zima sous forme d'un exposé Power Point « Biodiversité du Lac Zima »;

- atelier 2 : Construisons une station d'épuration ;

- atelier 3 : Éducation à l'environnement, tri et recyclage des déchets ;

- atelier 4 : Répertorier les espèces et les classifier, espèces menacées.

Un petit atelier d'arts plastiques est venu clore ces quatre ateliers : quelques collégiens ont été invités à réaliser des peintures illustrant les problématiques traitées lors des ateliers.

Par ailleurs, une exposition réalisée par les étudiants du Master susmentionné et présentant la richesse ornithologique du Lac Zima était disponible dans le hall d'entrée du collège. Cette exposition séjourne actuellement sur le site avant de devenir itinérante (autres sites et autres clubs du réseau RNCST).
La journée du 5 juin 2011, Journée mondiale de l’Environnement, a été pleinement consacrée à la sortie de découverte et d'éducation à l'environnement sur le site du lac Zima. Elle a d'abord consisté en une présentation générale du site par un expert ornithologue de l'université Cadi Ayyad de Marrakech puis, en une observation des populations d'oiseaux présentes sur le site. Les participants ont eu des explications détaillées sur le fonctionnement de cet écosystème, sur les dangers qu'il encourt et sur la manière de le protéger. En particulier, les experts ont insisté sur la relation entre la venue des oiseaux migrateurs et les éléments nutritifs présents dans la composition des eaux du lac. De ce point de vue, l'extraction du sel du lac Zima, si elle n'est pas maîtrisée, représente un réel danger pour la présence de ces espèces, dont certaines figurent sur la liste mondiale des espèces rares et menacées.

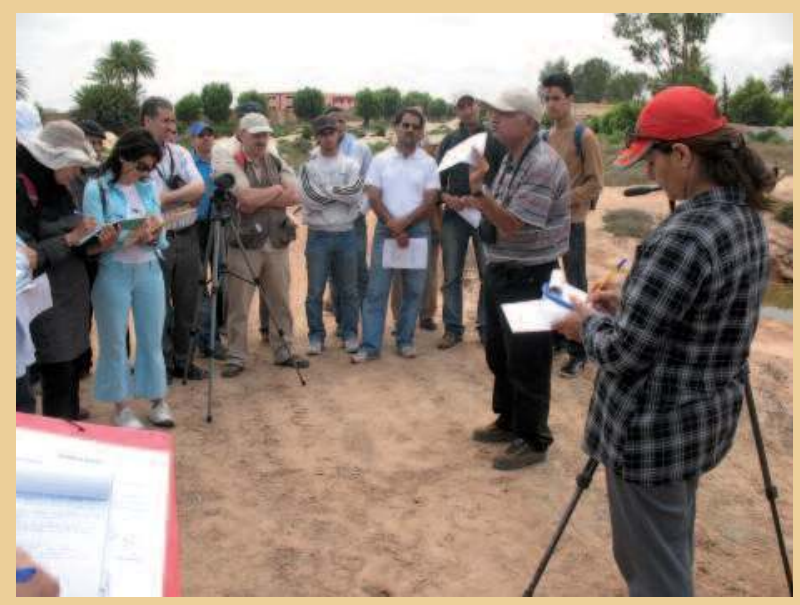

Exposé sur le fonctionnement de l'écosystème du lac Zima abritant des populations d'oiseaux en danger, juin 2011. ○) Muséum d'Histoire naturelle de Marrakech/Mohamed Ghamizi 
Dans une ferme volonté de pérennisation de ces actions, l'initiatrice de ces ateliers CST en milieu rural a fondé avec des collègues enseignants-chercheurs, à la faculté des Sciences Ain Chock de Casablanca, le club universitaire « Science citoyenne » membre du RNCST. L'une des activités centrales de ce club est le concours annuel «Sciences et Expériences » invitant les étudiants candidats à proposer un montage expérimental illustrant un phénomène scientifique grand public. Beaucoup de ces expériences, toujours simples mais traitées de manière originale, sont transférables en milieu rural au prix d'une adaptation. Ainsi, ce concours a été à l'origine de nouveaux ateliers transférés en milieu rural et traitant de thématiques diverses : énergies renouvelables, filtres plantés...

L'investissement du milieu rural se poursuit dans de nouveaux projets dans le cadre du RNCST avec le concours des clubs dont la thématique implique de fortes actions sur le terrain. C'est ainsi que dans le cadre du projet Transavia Solid'Air (14) pour l'environnement, dont le RNCST est lauréat à travers trois de ses clubs (Science citoyenne, Association nature et patrimoine et muséum d'Histoire naturelle de Marrakech), deux sites d'importance écologique ont été retenus pour mener des actions d'éducation à l'environnement : le Lac Zima (Région de Marrakech) et le site d'Intérêt Biologique et Écologique (SIBE) de l'embouchure de la Moulouya (Région d'Oujda).

Pilotée par le muséum d'Histoire naturelle de Marrakech (MHNM), la première action du projet, consacrée au lac Zima (voir encadré page précédente), a eu lieu les 4 et 5 juin 2011, faisant suite aux journées scientifiques organisées les 2 et 3 juin par le MNHM à Marrakech sur le thème « Les musées scolaires et le rôle des collections dans la didactique des Sciences de la Vie et de la Terre (SVT) » et coïncidant avec la journée mondiale de l'environnement. Outre les étudiants du Master Ingénierie écologique et Méthodologies d'Analyse et de Gestion de la Biodiversité (faculté des Sciences Semlalia, université Cadi Ayyad, Marrakech) encadrés par les membres du MHNM, divers acteurs CST régionaux ont pris part à cette action : enseignants-chercheurs spécialistes, professeurs des collèges et du secondaire, professeurs des écoles, membres du mouvement associatif.

\section{Conclusion}

Le développement de la CST au Maroc, souhaité officiellement par tous les acteurs - l'État en premier lieu -, exige une multitude de décisions et d'actions dont la mention n'entre pas dans le cadre de cet article (16). Celles-ci apparaissent toutefois en filigrane des insuffisances à la fois structurelles et en matière d'initiatives que nous avons soulignées plus haut. Par ailleurs, au Maroc comme ailleurs dans le monde, il est primordial de renforcer l'enseignement des sciences à l'école et dans les collèges. Un tel programme existe, sans résultats probants à l'heure actuelle et sans la participation des universités.

Mais, en dehors des mesures matérielles et organisationnelles qui pourraient être prises sous l'impulsion de l'État, un fait d'ordre psychologique nous semble d'une importance cruciale. Il faut arriver à briser l'indifférence qui règne autour de la science et des scientifiques dans la société marocaine. Cette indifférence a pour réciproque, il faut bien le reconnaître, la déconnexion que le scientifique marocain auto-entretient vis-à-vis de son environnement humain. Il est temps d'en sortir.

Dans un autre registre, en tant qu'acteurs de la CST et s'agissant de nos actions et de notre expérience sur le terrain relatées à travers les deux exemples présentés, il est clair que le défi actuel est d'assurer la permanence, la pérennité et la diffusion de ces actions à plus grande échelle. Ceci ne peut se faire que dans le cadre d'un réseautage associatif régional s'appuyant sur les collectivités locales et territoriales et les réseaux scolaires, dont l'exemple existe dans le Maroc oriental (17). Les réseaux nationaux, comme le nôtre, pourraient alors assumer le rôle de formateur et de fournisseur de ressources.

\section{Notes}

(1) Sabrie, M.-L. Promouvoir la culture scientifique et technique en Afrique, la Lettre de l'OCIM, n¹28, 2010, pp. 5-11.

(2) Enquête CERLIS : Séverine Dessajan et Elsa Ramos, Centre de Recherche sur les liens Sociaux (CERLIS, UMR 8070 CNRS/université Paris V), www.latitudesciences.ird.fr, rubriques PCST/ études et articles (3) Séminaires internationaux PCST : Oujda 2006, Ouagadougou 2008, Marseille 2009. www.latitudesciences.ird.fr, rubriques PCST/actions (4) Données de la Banque Mondiale sur le Maroc : http://donnees.banquemondiale.org/pays/maroc

(5) Rapport PNUD 2010

(6) Bensalah, A. How Morocco faces the challenge of science literacy. SciDev net, 11 février 2011 : www.scidev.net/fr/opinions/stimuler-la-culture-scientifique-au-maroc.html

(7) Séminaire international PCST : Oujda 2006, Expériences et pratiques de la culture scientifique et technique au Sud, du 25 au 27 janvier 2006 au Maroc. www.latitudesciences.ird.fr, rubriques PCST/actions 
(8) Colloque international La Culture Scientifique et Technique an Maroc: esquisse d'un état des lieux et perspectives d'une politique nationale à la lumière des expériences étrangères, 26 au 28 octobre 2009 Rabat, Maroc.

(9) Initiative nationale pour le Développement humain au Maroc : www.indh.ma.

(10) Projets lauréats «Association les Rangs d’Honneur », PCST2 «Apport et diffusion d'éléments scientifiques chez les jeunes du milieu rural »; PCST7 « Pour une meilleure diffusion de la culture scientifique au Maroc », www.latitudesciences.ird.fr rubrique PCST/appel à projets.

(11) Mikou, A. Quelle culture scientifique pour les jeunes du milieu rural marocain? Actes des Journées internationales sur la communication, l'éducation et la culture scientifiques, techniques et industrielles, Chamonix 2008.

(12) Le Monde.fr : http://lemonde-educ.blog.lemonde.fr/201 1/05/25 /afaf-mikou-la-chercheuse-qui-amene-la-mer-aux-petits-marocains-deszones-arides.
(13) Académie des Sciences : www.academie-sciences.fr/presse/communique/prixpurkwa_280411.

(14) RNCST, Projet lauréat en 2010 du programme Solid'air Transavia «sensibilisation aux enjeux de la biodiversité et du développement durable », www.solidair-transavia.com

(15) Ramsar : Convention internationale ratifiée en 1971 à Ramsar en Iran et instituant une liste internationale de zones humides à protéger.

(16) Bensalah, A. Introduction au concept de culture scientifique et technique : aspects succincts ressortis de l'expérience occidentale, Académie Hassan II des sciences et des techniques, bulletin $\mathrm{n}^{\circ} 5$, juin 2009.

(17) CEPO : Consortium Environnement et Patrimoine de l'Oriental 